\title{
A primer on diffusion tensor imaging of anatomical substructures
}

\author{
Alexandre F. M. DaSilva, D.D.S., D.M.Sc., David S. Tuch, Ph.D., \\ Mette R. Wiegell, Ph.D., and Nouchine Hadjikhani, M.D. \\ Martinos Center for Biomedical Imaging, Massachusetts General Hospital, NMR Center, Harvard \\ Medical School, Charlestown, Massachusetts
}

\begin{abstract}
In this article, the authors review the application of diffusion tensor (DT) magnetic resonance (MR) imaging to demonstrate anatomical substructures that cannot be resolved by conventional structural imaging. They review the physical basis of DT imaging and provide illustrative anatomical examples. The DT imaging technique measures the self-diffusion, or random thermal motion, of the endogenous water in nerve tissue. Because of the preferred diffusion of water molecules along the nerve fiber direction, DT imaging can measure the orientation of the neural fiber structure within each voxel of the MR image. The fiber orientation information yielded by DT imaging provides a new contrast mechanism that can be used to resolve images of anatomical substructures that cannot otherwise be visualized using conventional structural imaging. The authors illustrate how DT imaging can resolve individual pathways in the brainstem as well as individual nuclei of the thalamus and conclude by describing potential applications in neurosurgery.
\end{abstract}

KEY WORDS - diffusion tensor imaging - trigeminal nerve $\quad$ brainstem - thalamus

Since the advent of DT imaging in the early 1990 s, 2,20,26 the technique has generated a tremendous amount of interest in the clinical and laboratory domains. This modality measures the random thermal displacement (that is, Browian motion) of molecules in tissue, typically water molecules. Two aspects of DT imaging render the modality very powerful. First, the microscopic lengthscale of water diffusion in tissue gives DT imaging microscopic spatial sensitivity. Second, in fibrous tissues, such as cerebral white matter or organized gray matter, the diffusion is anisotropic, that is, orientation dependent. The orientation dependence of the diffusion signal enables DT imaging to measure the fiber orientation within each voxel of the image.

\section{DIFFUSION TENSOR IMAGING}

The relative contribution of the various cellular and subcellular components to diffusion anisotropy is not completely understood and represents an active area of research. Myelin is thought to be one of the most significant contributors to diffusion anisotropy because of the diffusion barrier imposed by the lipid bilayer. Myelin, however, is not an obligatory requirement for diffusion anisotropy. Beaulieu and Allen ${ }^{4}$ showed that the trigemi-

Abbreviations used in this paper: DT = diffusion tensor; MPRAGE = magnetization-prepared rapid-acquisition gradientecho; MR = magnetic resonance; MS = multiple sclerosis. nal, optical, and olfactory nerves in garfish presented significant anisotropy equivalent to humans despite the nonmyelination of the olfactory nerve, suggesting that the dense packing of axons, as well as the axonal structures such as membranes, microtubules, neurofilaments, and microfilaments may contribute to the anisotropy. In addition to structural components, there are other metabolic mechanisms that may contribute to the diffusion anisotropy, including axonal transport and cytosolic streaming. ${ }^{3}$

For isotropic, orientation-independent diffusion, such as that occurring in cerebrospinal fluid, the diffusion can be described in terms of the apparent diffusion coefficient.

For anisotropic, orientation-dependent diffusion, however, the diffusion is described through the diffusion tensor "D." The direction of greatest diffusion is given by the principal eigenvector " $\mathrm{e}_{1}$ " of the diffusion tensor. As the direction of greatest diffusion, the principal eigenvector parallels the local fiber direction within each voxel. The principal eigenvector maps, which display the fiber orientation, can be visualized as vector-field or colorcoded maps. To visualize the fiber direction map in the context of a conventional structural image, the fiber map can be registered to and superimposed on a structural image, such as a high-resolution $\mathrm{T}_{1}$-weighted MR image. In Figure 1, a mosaic of color-coded DT image maps superimposed on the MPRAGE structural image obtained in the same patient is shown. The DT image map was registered to the MPRAGE images by using the Functional Linear Imaging Registration Tool, ${ }^{17}$ which is distributed 
by the Oxford Centre for Functional Magnetic Resonance Imaging of the Brain. In registering the DT image to the structural images, the tensors were also reoriented to account for the different slice orientations of the two different images. The fiber orientation information provided by color-coded DT imaging maps can be used to differentiate among nerve fiber pathways. Further, the fiber orientation maps can delineate anatomical substructures based on their distinct fiber architecture.

Although a promising neuroimaging technique, there are few caveats to consider when using this modality. In regions of intravoxel fiber crossing, the fiber vector represents a partial volume mean and therefore does not reflect the underlying fiber structure. To resolve this partial volume effect, novel approaches such as high angular resolution diffusion imaging are being developed. ${ }^{13,30}$ Another restriction of DT imaging is sensitivity to motion, including pulsatile cardiac and respiratory motion. Additionally, echo planar imaging- based DT imaging is hindered by susceptibility distortions at the proximity of the skull base and air-filled spaces, such as in the case of the trigeminal nerve root and brainstem. Such susceptibility distortions can be eliminated using non-echo planar imaging sequences, which improve localization of the cranial nerves. ${ }^{18}$
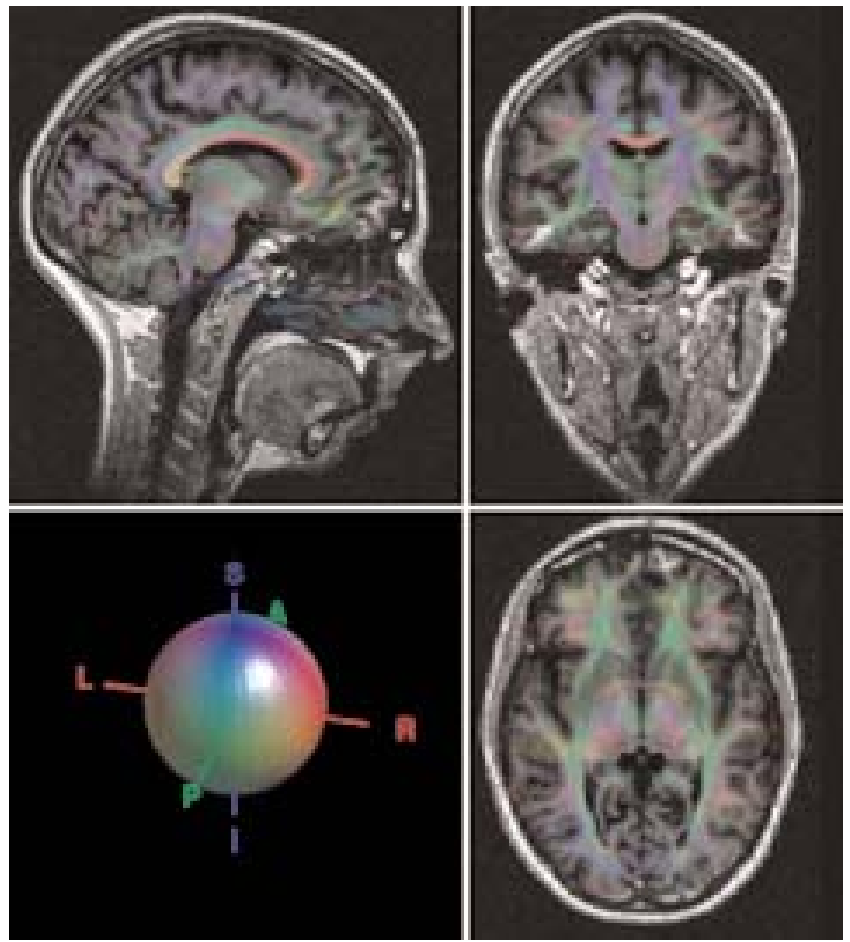

Fig. 1. Color-coded DT image map superimposed on a highresolution anatomical image. The DT image map is rendered in the sagittal (upper left), coronal (upper right), and axial (lower right) planes. The color coding depicts the local fiber orientation (that is, the principal eigenvector of the diffusion tensor) with red indicating mediolateral, green anteroposterior, and blue superoinferior. The color coding is also indicated by the red-green-blue sphere (lower left). The DT imaging data were collected with a twice-refocused balanced echo sequence $\left(b=700 \mathrm{~second} / \mathrm{mm}^{2}\right.$, isotropic 1.8 $\mathrm{mm}$ resolution). The MPRAGE anatomical image was acquired using isotropic 1-mm resolution.

\section{Anatomical Substructure in the Brainstem and Thalamus}

To illustrate the ability of DT imaging to demonstrate anatomical substructure, we provide examples from the brainstem and the thalamus.

Brainstem. The brainstem contains several ascending and descending tracts between the spinal cord and the forebrain, and it relays afferent and efferent neuronal fibers through several nuclei. These nuclei also receive inputs from the cranial nerves. The trigeminal nerve can be demonstrated on DT imaging, along the projection of the nerve root from the trigeminal ganglion to the brainstem (Fig. 2). At the insertion to the brainstem, the trigeminal fibers connect to the mesencephalic, motor, main sensory, and spinal trigeminal brainstem nuclei. ${ }^{9,27}$ Using DT imaging, Mamata and colleagues ${ }^{22}$ were able to reveal the subsequent tracts, including the trigeminothalamic and spinothalamic tracts, conveying the sensory information to the forebrain. In another study, using three-dimensionalguided quantitative and tracking analysis, Stieltjes, et al., ${ }^{29}$ distinguished the corticospinal tract, the medial lemniscus, and the superior, medial, and inferior cerebellar peduncles.

Potential Applications in the Brainstem. The ability to resolve images of anatomical substructure in the brainstem with DT imaging will help localize surgical targets with respect to specific tracts or nuclei. For example, DT imaging may be able to identify the trigeminal subnucle-

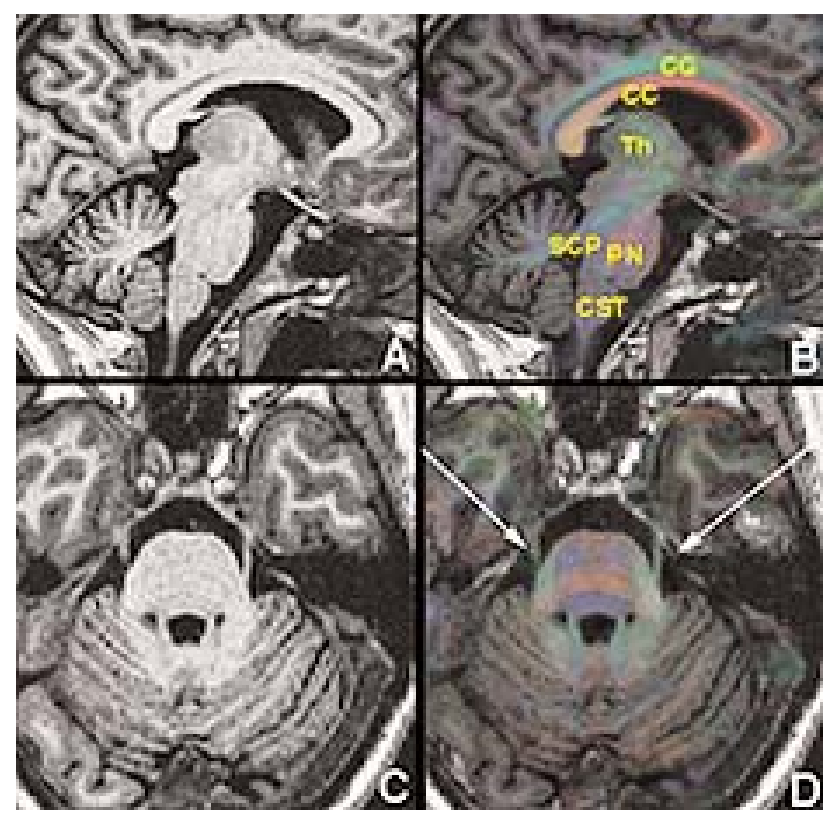

Fig. 2. Color-coded DT image map superimposed on high-resolution anatomical image. The directional color coding is the same as that in Fig. 1. A and B: A parasagittal structural image (A) and the corresponding DT image map (B) from the brainstem are shown. C and D: An axial structural image (C) and the corresponding DT image map (D) are shown for comparison. The trigeminal roots are indicated by the white arrows. The superior cerebellar peduncle pathways (green) can also be seen on the posterior aspect of the pons. $\mathrm{CC}=$ corpus callosum; $\mathrm{CG}=$ cingulate gyrus; $\mathrm{CST}=$ corticospinal tract; $\mathrm{PN}=$ pontine nucleus; $\mathrm{SCP}=$ superior cerebellar peduncle; $\mathrm{Th}=$ thalamus . 
us caudalis, which is a target for ablation in cases of trigeminal chronic pain. In cases of trigeminal neuralgia, in which the origin is believed by some to be vascular compression of the trigeminal root at the level of the pons, ${ }^{21}$ DT imaging may be able to depict the compression-induced fiber displacement. Evaluation of the affected tissue obtained from biopsy samples of the nerve root obtained during microvascular decompression in trigeminal neuralgia has shown different levels of axonopathies and myelin irregularities in addition to proliferation of collagen. ${ }^{11}$ Other than vascular compression, tumors and MS lesions are known to reproduce symptoms of typical and atypical trigeminal neuralgias and dysfunctions depending on their location in the trigeminal sensory system. ${ }^{1,7,24}$ Therefore, it is reasonable to predict that the measurement and mapping of changes in the diffusion anisotropy for different portions of the trigeminal system can supply additional information pertaining to the differential diagnosis of trigeminal neuropathies.

In diagnosing trigeminal neuralgia, it is essential to exclude a diagnosis of $\mathrm{MS},{ }^{25}$ especially in young patients. The authors of some studies have shown a decrease in the diffusion anisotropy and an increase in the mean diffusivity of MS lesions of different phases, including relapsing-remitting and progressive phases. ${ }^{8,12}$ Because active MS lesions may induce distal degenerative changes in white matter not conventionally detected on MR imaging 5 (normal-appearing white matter), DT imaging can be used to demonstrate the real extension of the axonal damage.

Thalamus. In the thalamus, pathways responsible for sensory, motor, and cognitive processing are organized into distinct nuclei. These nuclei can be differentiated based on their distinct histological features but cannot be distinguished on conventional MR images. Recently, Wiegell and colleagues ${ }^{31}$ have shown that DT imaging can resolve individual thalamic nuclei based on their characteristic fiber orientations. Using an automated clustering algorithm, they extracted 14 hemispheric thalamic nuclei and subnuclei. The anatomical parcellation results corresponded with previous histology-based descriptions of the nuclei. Figure 3 provides a comparison of an axial structural MPRAGE image and a DT imaging image at the level of the thalamus. Whereas little discernible thalamic substructure can be seen on the MPRAGE image, the DT image reveals the nuclear organization based on fiber orientation.

Potential Applications in the Thalamus. Localization of thalamic nuclei based on fiber architecture will provide for more accurate surgery in the thalamic region. This would be especially useful for preoperative planning of ablative or electrical stimulation neurosurgeries in which different substructures are targeted, such as the ventral posterior nucleus for chronic pain disorders, ${ }^{19}$ subthalamic nuclei for Parkinson disease ${ }^{10}$ and epilepsy, ${ }^{6}$ posterior hypothalamus for cluster headache, ${ }^{14}$ and internal capsule for obsessive-compulsive disorder. ${ }^{15}$

\section{Other Applications of DT Imaging in Preop Planning}

The sensitivity of DT imaging to fiber orientation and density can facilitate the differential diagnosis of tumors. In particular, DT imaging has been used to quantify massinduced fiber reorientation and/or compression. ${ }^{16} \mathrm{Ad}-$ ditionally, DT imaging can demonstrate compression effects due to vascular malformations. Wieshmann and colleagues $^{33}$ reported that DT images demonstrated the displacement of nearby healthy structures due to a lowgrade glioma in the frontal lobe without degeneration of the fibers, which was in agreement with the clinical findings. In another report of two different cases of anaplastic astrocytoma, the DT color-coded images were used to characterize displacement and infiltration of nearby white matter. ${ }^{23}$ In a larger study of nine patients with high-grade cerebral glioblastoma, Sinha and colleagues ${ }^{28}$ reported that mean diffusion, but not diffusion anisotropy, was significantly helpful for the delineation of the tumor margins, its consequent edema, and the surrounding healthy white matter. The same results were achieved in patients with brain tumor after dexamethasone treatment, and a significant decrease in the mean diffusion of the tumor and edema area was also found. Although the authors of another DT imaging study identified reduced anisotropy in different brain tumors, including meningioma, hypothalamic hamartoma, and glioma compared with control individuals, ${ }^{32}$ additional studies are needed to enhance the power of DT imaging to define precisely the boundaries between tumors and healthy tissue.

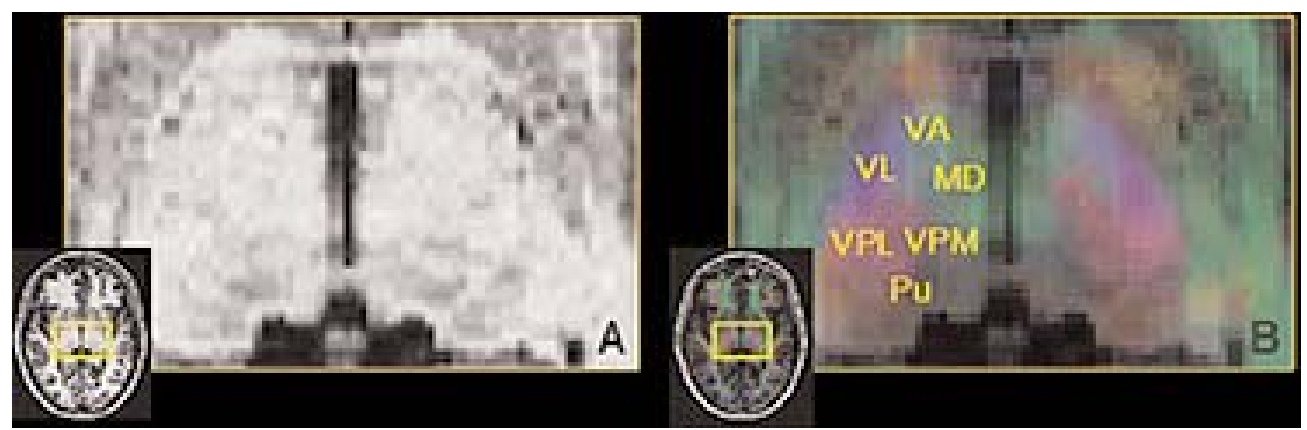

Fig. 3. An axial MPRAGE structural image (A) and the corresponding color coded DT image (B) are shown for comparison. The directional color coding is the same as that in Fig. 1. The region of interest is taken from the yellow box shown in the whole slice image in the lower left corner. On the MPRAGE, the thalamus appears homogeneous, whereas the DT image map demonstrates significant substructure. The thalamic nuclei have been labeled according to their anatomical position and fiber orientation. $\mathrm{MD}=$ mediodorsal; $\mathrm{Pu}=$ pulvinar; $\mathrm{VA}=$ ventral anterior; $\mathrm{VL}=$ ventrolateral; $\mathrm{VPL}=$ ventral posterolateral; $\mathrm{VPM}=$ ventral posteromedial 


\section{CONCLUSIONS}

Recent advances in DT imaging promise a revolution in MR imaging for presurgical planning. This modality will increase our capacity to identify anatomical substructures that cannot be visualized by other existing neuroimaging tools. Furthermore, as we have illustrated here, visualization of DT fiber direction maps in the context of structural images provides enhanced localization of anatomical substructures. Last, the microscopic sensitivity of DT imaging will benefit the noninvasive assessment of the microstructural aspects of brain lesions.

\section{References}

1. Al-Mefty O, Ayoubi S, Gaber E: Trigeminal schwannomas: removal of dumbbell-shaped tumors through the expanded Meckel cave and outcomes of cranial nerve function. J Neurosurg 96:453-463, 2002

2. Basser PJ, Mattiello J, LeBihan D: MR diffusion tensor spectroscopy and imaging. Biophys J 66:259-267, 1994

3. Beaulieu C: The basis of anisotropic water diffusion in the nervous system - a technical review. NMR Biomed 15:435-455, 2002

4. Beaulieu C, Allen PS: Determinants of anisotropic water diffusion in nerves. Magn Reson Med 31:394-400, 1994

5. Bjartmar C, Wujek JR, Trapp BD: Axonal loss in the pathology of MS: consequences for understanding the progressive phase of the disease. J Neurol Sci 206:165-171, 2003

6. Chabardes S, Kahane P, Minotti L, et al: Deep brain stimulation in epilepsy with particular reference to the subthalamic nucleus. Epileptic Disord (Suppl 3) 4:83-93, 2002

7. Cheng TM, Cascino TL, Onofrio BM: Comprehensive study of diagnosis and treatment of trigeminal neuralgia secondary to tumors. Neurology 43:2298-2302, 1993

8. Ciccarelli O, Werring DJ, Barker GJ, et al: A study of the mechanisms of normal-appearing white matter damage in multiple sclerosis using diffusion tensor imaging - evidence of Wallerian degeneration. J Neurol 250:287-292, 2003

9. DaSilva AF, Becerra L, Makris N, et al: Somatotopic activation in the human trigeminal pain pathway. J Neurosci 22: 8183-8192, 2002

10. Deuschl G, Wenzelburger R, Kopper F, et al: Deep brain stimulation of the subthalamic nucleus for Parkinson's disease: a therapy approaching evidence-based standards. J Neurol (Suppl 1) 250:43-46, 2003

11. Devor M, Govrin-Lippmann R, Rappaport ZH: Mechanism of trigeminal neuralgia: an ultrastructural analysis of trigeminal root specimens obtained during microvascular decompression surgery. J Neurosurg 96:532-543, 2002

12. Filippi M, Cercignani M, Inglese M, et al: Diffusion tensor magnetic resonance imaging in multiple sclerosis. Neurology 56:304-311, 2001

13. Frank LR: Characterization of anisotropy in high angular resolution diffusion-weighted MRI. Magn Reson Med 47: 1083-1099, 2002

14. Franzini A, Ferroli P, Leone M, et al: Stimulation of the posterior hypothalamus for treatment of chronic intractable cluster headaches: first reported series. Neurosurgery 52:1095-1101, 2003

15. Gabriels L, Cosyns P, Nuttin B, et al: Deep brain stimulation for treatment-refractory obsessive-compulsive disorder: psychopathological and neuropsychological outcome in three cases. Acta Psychiatr Scand 107:275-282, 2003
16. Holodny AI, Schwartz TH, Ollenschleger M, et al: Tumor involvement of the corticospinal tract: diffusion magnetic resonance tractography with intraoperative correlation. J Neurosurg 95:1082, 2001

17. Jenkinson M, Bannister P, Brady M, et al: Improved optimization for the robust and accurate linear registration and motion correction of brain images. Neuroimage 17:825-841, 2002

18. Koch MA, Glauche V, Finsterbusch J, et al: Distortion-free diffusion tensor imaging of cranial nerves and of inferior temporal and orbitofrontal white matter. Neuroimage 17:497-506, 2002

19. Kumar K, Toth C, Nath RK: Deep brain stimulation for intractable pain: a 15-year experience. Neurosurgery 40:736-747, 1997

20. Le Bihan D: Molecular diffusion nuclear magnetic resonance imaging. Magn Reson Q 7:1-30, 1991

21. Love S, Coakham HB: Trigeminal neuralgia: pathology and pathogenesis. Brain 124:2347-2360, 2001

22. Mamata H, Mamata Y, Westin CF, et al: High-resolution line scan diffusion tensor MR imaging of white matter fiber tract anatomy. AJNR 23:67-75, 2002

23. Mori S, Frederiksen K, van Zijl PC, et al: Brain white matter anatomy of tumor patients evaluated with diffusion tensor imaging. Ann Neurol 51:377-380, 2002

24. Nakashima I, Fujihara K, Kimpara T, et al: Linear pontine trigeminal root lesions in multiple sclerosis: clinical and magnetic resonance imaging studies in 5 cases. Arch Neurol 58: 101-104, 2001

25. Nurmikko TJ, Eldridge PR: Trigeminal neuralgia-pathophysiology, diagnosis and current treatment. Br J Anaesth 87: 117-132, 2001

26. Pierpaoli C, Righini A, Linfante I, et al: Histopathologic correlates of abnormal water diffusion in cerebral ischemia: diffusion-weighted MR imaging and light and electron microscopic study. Radiology 189:439-448, 1993

27. Sessle BJ: Acute and chronic craniofacial pain: brainstem mechanisms of nociceptive transmission and neuroplasticity, and their clinical correlates. Crit Rev Oral Biol Med 11: 57-91, 2000

28. Sinha S, Bastin ME, Whittle, IR, et al: Diffusion tensor MR imaging of high-grade cerebral gliomas. AJNR 23:520-527, 2002

29. Stieltjes B, Kaufmann WE, van Zijl PC, et al: Diffusion tensor imaging and axonal tracking in the human brainstem. Neuroimage 14:723-735, 2001

30. Tuch DS, Reese TG, Wiegell MR, et al: High angular resolution diffusion imaging reveals intravoxel white matter fiber heterogeneity. Magn Reson Med 48:577-582, 2002

31. Wiegell MR, Tuch DS, Larsson HBW, et al: Automatic segmentation of thalamic nuceli from diffusion tensor magnetic resonace imaging. Neuroimage 19:391-401, 2003

32. Wieshmann UC, Clark CA, Symms MR, et al: Reduced anisotropy of water diffusion in structural cerebral abnormalities demonstrated with diffusion tensor imaging. Magn Reson Imaging 17:1269-1274, 1999

33. Wieshmann UC, Symms MR, Parker GJ, et al: Diffusion tensor imaging demonstrates deviation of fibers in normal appearing white matter adjacent to a brain tumour. J Neurol Neurosurg Psychiatry 68:501-503, 2000

Manuscript received May 30, 2003.

Accepted in final form June 13, 2003.

Address reprint requests to: Nouchine Hadjikhani, M.D., Martinos Center for Biomedical Imaging, NMR Center, Building 36, First Street, Room 417, Charlestown, Massachusetts 02129. email: nouchine@nmr.mgh.harvard.edu. 